\title{
Automatic Generation of Funny Cartoons Diary for Everyday Mobile Life
}

\author{
Injee Song, Myung-Chul Jung, and Sung-Bae Cho \\ Computer Science Department, Yonsei University \\ 134 Shinchon-dong, Sudaemoon-ku, Seoul 120-749, South Korea \\ \{schunya, mcjung, sbcho\}@sclab.yonsei.ac.kr
}

\begin{abstract}
The notable developments in pervasive and wireless technology enable us to collect enormous sensor data from each individual. With contextaware technologies, these data can be summarized into context data which support each individual's reflection process of one's own memory and communication process between the individuals. To improve reflection and communication, this paper proposes an automatic cartoon generation method for fun. Cartoon is a suitable medium for the reflection and the communication of one's own memory, especially for the emotional part. By considering the fun when generating cartoons, the advantage of the cartoon can be boosted. For the funnier cartoon, diversity and consistency are considered during the cartoon generation. For the automated generation of diverse and consistent cartoon, context data which represent the user's behavioral and mental status are exploited. From these context information and predefined user profile, the similarity between context and cartoon image is calculated. The cartoon image with high similarity is selected to be merged into cartoon cuts. Selected cartoon cuts are arranged with the constraints for the consistency of cartoon story. To evaluate the diversity and consistency of the proposed method, several operational examples are employed.
\end{abstract}

\section{Introduction}

Personal computing and World Wide Web fundamentally change each individual's communication environments at home and the office. People in the world present their thought to a large number of anonymous people by posting articles, images and videos on their personal web page and web log. Pervasive computing environments will not only change and enhance our office environment, but also change our communication pattern in the relationship with family members, friends and business partners in the everyday life. Through the sensors such as GPS, visual and audio sensors in the pervasive computing environment, we can collect log data from each individual's daily life. These logged sensor data can be employed to infer user's behavior, emotion, and surrounding by applying context-aware techniques [1]. Such inferred context data are useful in summarizing an individual's daily life. Like a personal diary, summarized context is helpful in reflecting one's own memory and in strengthening the communication process. Even though multimedia representation of the context like images and videos could be more ambiguous than simple text, multimedia is 
more effective to represent and share impressions about personal experiences [2]. Particularly, cartoon can effectively express personal emotion. Especially, funny cartoon enhances the impression of personal experiences. To make funnier contents, contents designers make various patterns in the contents while considering users' cognitive acceptability [3].

For the automated generation of funny cartoon, this paper focuses on the diversity of cartoon images and the consistency of cartoon story. Various patterns in the cartoon can be made by diverse cartoon images, and the readers' cognitive acceptance is considered using consistency constraints. For the automatic cartoon generation, we compose character and background images from the user profile and landmark context events. To find appropriate character and background images to the landmark events, semantic similarities between landmarks and images are computed. After composing, generated cartoon cuts are reorganized using consistency constraints to keep the consistency of cartoon story. The formal description of the problem is the following. Given a tuple $L$ of $n$ landmark events, a cartoon story $C S_{k}$ is a tuple $C S_{k}=$ $\left\{C C_{k 1}, C C_{k 2}, \ldots, C C_{k n}\right\}$ where

- $L=\left\{\right.$ event $_{1}$, event $_{2}, \ldots$, event $\left._{n}\right\}$ is a tuple of landmark events in which event $t_{x}$ is the $x^{\text {th }}$ event that is extracted from daily context data;

- $C C_{k m}=\left\{\right.$ CartoonImage $_{k m 1}$, CartoonImage $_{k m 2}, \ldots$, CartoonImage $\left._{k m h}\right\}$ is a tuple of cartoon images that describe the cartoon cut which is the most feasible to the $m^{\text {th }}$ event and consists of $h$ different types of cartoon images, i.e., character image, background image, etc.

\section{Related Works}

With the development of pervasive and mobile technology, many context aware researches are going on. R. Miikkulainen proposed the episodic memory that categorizes stories and recovers script-based memories by hierarchal SOM (Self-organizing Feature Maps) [4]. E. Horvitz made a Bayesian network representation of human cognitive behavior [5]. He also designed a learning model that found out critical events from online calendar software. Together with context-aware researches, many researchers have been studying about how to represent these context data effectively. B. Viégas of MIT media laboratory created two visualizations from email archives. One highlighted social networks and the other depicted the temporal rhythms of interactions with individuals [6]. ATR institute in Japan developed ComicDiary [7] as subsystem of C-MAP project which was visitor guidance system for exhibition. ComicDiary organizes scenario using story stream method and composes the cartoon image and text using template. Because our research focuses on the diversity of cartoon composition, methods using predefined templates will generate only limited set of cartoons.

When we compose character and background images with user's context data, there is a process that finds relevant images for context data. For this process, we can utilize image retrieval researches [8]. Generally, there are two kinds of retrieval methods. One is QBK (query-by-keywords) and the other is QBE (query-by-example). When we compose a cartoon image, QBK approach is more applicable. M. Inoue suggested image retrieval methods for lightly annotated images by using image similarities [9]. In 
the retrieval process, annotations in the images can be used as query keywords. In the sense that QBK requires to compute semantic similarities between keywords and the images, it can be compared to compose various web services with user's service request. To compose web services, Cardoso matches service object (SO) and service template (ST) in the web service searching process [10]. SO defines the real web service component that system wants to find, and ST defines the functions of web service that user requests. Matching process consists of two steps. At first, system looks for SO that is the most similar to ST using structural and functional similarities. After that, to match actual functions of SO and ST, semantic similarity between functions of SO and ST is utilized. Semantic similarity is computed by employing the numerical formula similar to Jaccard coefficient like in the following equation.

$$
S_{i j}=\frac{p}{p+q+r} .
$$

$S_{i j}=$ similarities between object $i$ and object $j$

$p=$ number of attributes that object $i$ and object $j$ all have

$q=$ number of attributes that object $i$ has, but object $j$ does not

$r=$ number of attributes that object $j$ has, but object $i$ does not

\section{Cartoon Generation Method}

Entire process of cartoon generation is shown in Fig. 1. It selects the character and background images in the similarity of the landmark events from context-aware module with user profile. In selection process, semantic similarity between images and landmark event is calculated based on the predefined annotations for the images. After selecting the cartoon cuts for each context data, they are organized into a story stream to make a plausible cartoon story.

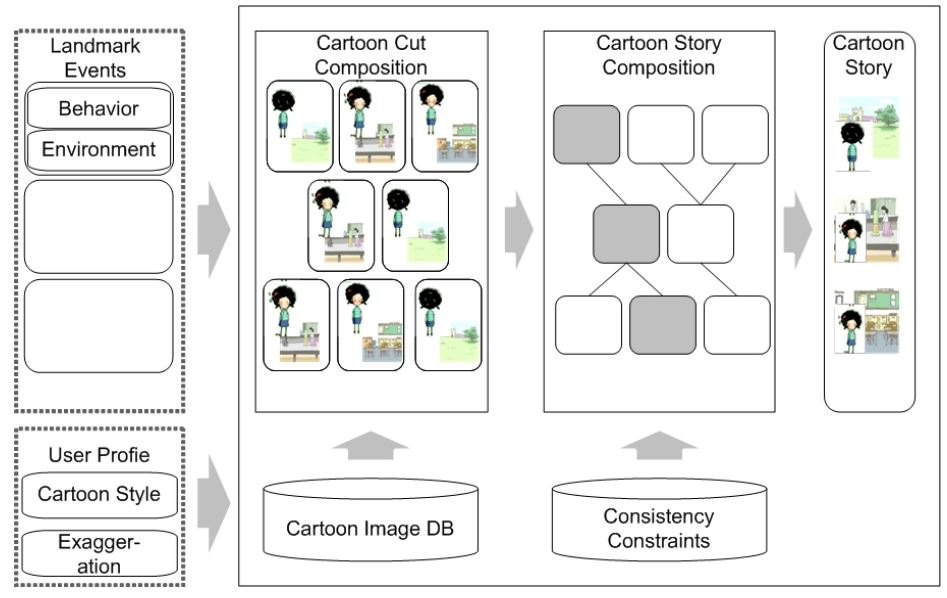

Fig. 1. Cartoon generation process 


\subsection{Landmark Events and User Profile}

For the automatic composition of cartoon images, landmark events and user profile are used as input data. A landmark event is a set event $x_{x}=(<$ Behavior $\rangle,<$ Environ$m e n t>$ ) is the $x^{\text {th }}$ landmark event in chronological order which describes the important event happened during the day consists of behavioral and environmental landmarks. Behavioral landmark $<$ Behavior $>$ is a set of keywords that describe user's action and emotional status. Environmental landmark <Environment $>$ is a set of keywords that describe the place where the event happens. User profile is a set of values that reflect the user's preference about the cartoon image. For example, preferred character style and preference about exaggerated cartoon image can be used as attribute.

\subsection{Cartoon Cut Composition}

A cartoon cut $C C_{k m}=\left\{\right.$ CharacterImage $_{k m}$, BackgroundImage $\left._{k m}\right\}$ is a tuple of cartoon images that describe the cartoon cut which is the $k^{\text {th }}$ feasible cartoon composition to the $m^{\text {th }}$ landmark event. To compose diverse cartoon cuts, we match landmark events and images in a flexible way. After defining images with the keywords from the same ontology which define landmark events, we match cartoon image and landmark event by the semantic similarity between them. By means of semantic similarity, we cannot only acquire various cartoon cuts from limited images but also easily expand the annotation of landmark events and cartoon images. At first, semantic similarity between the cartoon images and landmark events are calculated using the equation (2). From the images whose similarities are higher than certain threshold, the candidate list of cartoon cuts is generated. The threshold for image selection is defined in consideration of cartoon diversity and computational cost. Lower threshold will compose more diverse cartoon expression, but will require more computational cost for the consistency constraint violation check.

$$
\text { Similarity }\left(\text { event }_{m}, \text { image }_{k m h}\right)=\frac{\mid\left(p\left(\text { event }_{m}\right) \cup p(U P)\right) \cap p\left(\text { image }_{k m h}\right) \mid}{\mid p\left(\text { event }_{m}\right) \cup p(U P) \cup p\left(\text { image }_{k m h}\right) \mid} .
$$

Similarity $\left(\right.$ event $_{m}$, image $\left._{k m h}\right)=$ similarities between event $_{m}$ and image kmh $_{\text {in }}$ event $_{m}=$ the $m^{\text {th }}$ landmark event image $_{k m h}=$ cartoon image of type $h$ in the $k^{\text {th }}$ feasible cartoon cut composition for the $m^{\text {th }}$ landmark event

$U P=$ user profile

$p(X)=$ a set of attributes that object $X$ has

\subsection{Cartoon Story Composition}

After image selection process for cartoon cut composition, a number of cartoon cuts are acquired for each landmark event. These cartoon cut lists are organized into story stream to select a cartoon story from available stories. The story stream is a graph constructed by connecting cartoon cuts satisfying two conditions $m \neq n$ and a tuple $\{x$, $y\} \notin$ ConsitencyConstraints where 
- $C C_{k m}$ is the $k^{\text {th }}$ feasible cartoon cut composition for the $m^{\text {th }}$ landmark event;

- $C C_{j n}$ is the $j^{\text {th }}$ feasible cartoon cut composition for the $n^{\text {th }}$ landmark event;

- ConsistencyConstraints is a set of tuples that consist of the preceding relationship between behaviors or emotions. For the preceding relationship in consistency constraints, the consistency of the character's emotion and the character image style are considered. For an example, ("Very happy", "Very sad");

- $K(X)=p\left(\right.$ image $\left._{X 1}\right) \cup p\left(\right.$ image $\left._{X 2}\right) \cup \ldots \cup p\left(\right.$ image $\left._{X l}\right)$ is a union of keyword sets that describe images (image $_{X 1}$, , $^{\text {mage }} X_{X 2}, \ldots$, image $_{X l}$ ) in cartoon cut $X$;

- $x \in K\left(C C_{k m}\right)$ and $y \in K\left(C C_{j n}\right)$.

After constructing cartoon story stream, available cartoon stories are searched through the story stream graph using the process described in Fig. 2. One cartoon story among the candidates is randomly selected to be presented as the final cartoon.

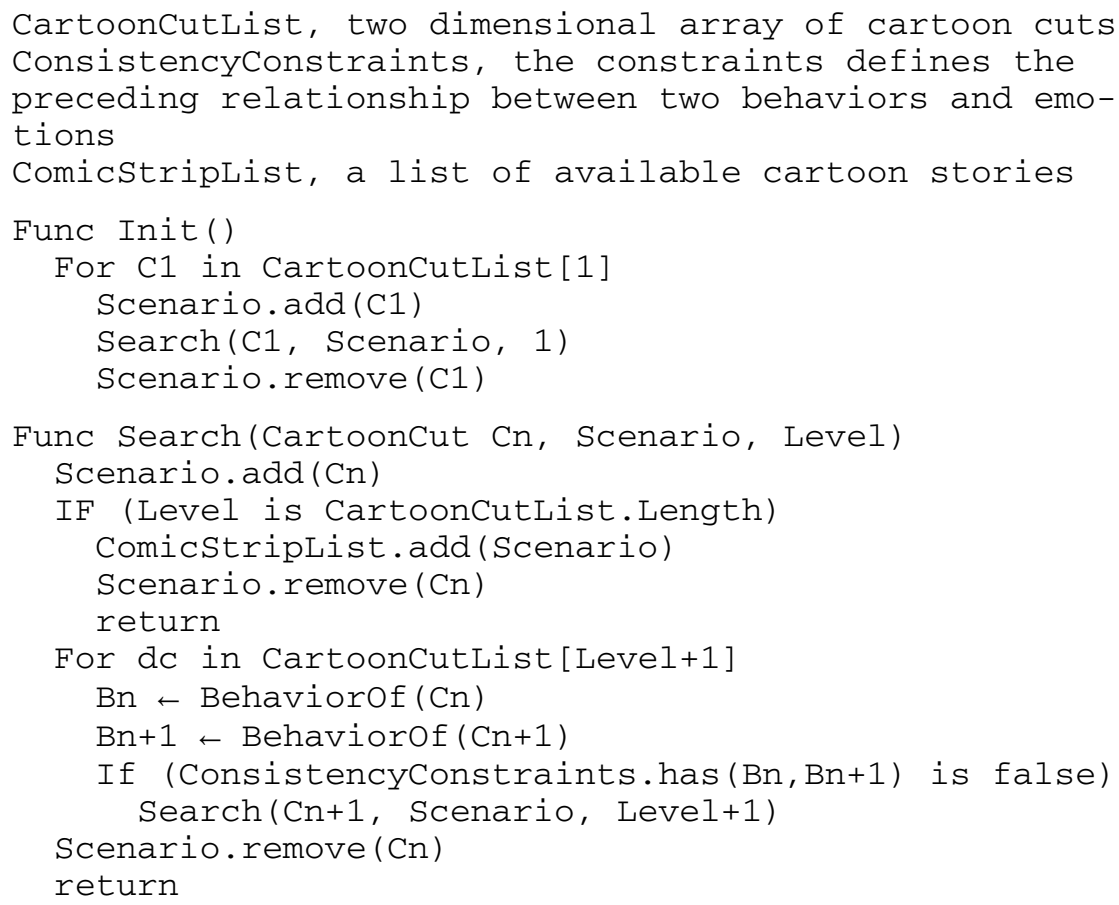

Fig. 2. Cartoon story search process

\section{Operational Examples}

\subsection{Scenario Description}

Because the fun of the composed cartoon story can be evaluated only in subjective measure, we evaluate the fun of the cartoon by evaluating the diversity and the consistency of the cartoon. To evaluate the generated cartoon story, we create sample 
Table 1. Landmark context examples

\begin{tabular}{lll}
\hline ID & Behavioral landmark & Environmental landmark \\
\hline 1 & Movement, MP3, Stand & Bus, Indoor \\
2 & Study, Take a class, Sit & University, Classroom, Indoor \\
3 & Eat, Korean Food, Sit & University, Dinning Room, Indoor \\
4 & Talk, Phone, Stand & University, Outdoor \\
5 & Cheer, Watch, Stand & Stage, Outdoor \\
6 & Drink, Beer, Sit, Cheer & Drinking House, Bar, Indoor \\
\hline
\end{tabular}

Table 2. Category of keywords to describe landmark events and cartoon images

\begin{tabular}{|c|c|c|c|}
\hline \multicolumn{3}{|c|}{ Category of keywords } & Examples (Number of total keywords) \\
\hline \multirow{8}{*}{ Behavior } & \multirow{7}{*}{ Action } & Basic & Stand, Sit, Phone, See, Kiss, ...(16) \\
\hline & & Movement & Work, Run, Car, Bus, Subway(5) \\
\hline & & At home & Cook, Bath, Shower, Makeup, ...(12) \\
\hline & & In work & Study, Paperwork, Presentation, ...(11) \\
\hline & & Entertainment & Dance, Sing, Game, ...(8) \\
\hline & & Sports & Tennis, Golf, Ski, Swim, Yoga, ...(9) \\
\hline & & Others & Umbrella, Shopping back, ...(7) \\
\hline & \multirow[t]{2}{*}{ Emotion } & & Happy, Sad, Angry, Surprised, ...(30) \\
\hline \multirow{7}{*}{ Environme } & & Indoors & Classroom, Cafeteria, Office, ... (45) \\
\hline & \multirow{2}{*}{ Place } & Midtown & Sidewalk, Shopping quarters, ... (21) \\
\hline & & Nature & Woods, Mountain, Beach, ...(38) \\
\hline & & Imaginary & Heaven, Hell, Space, ...(35) \\
\hline & \multicolumn{2}{|l|}{ Weather } & Sunny, Cloudy, Rainy, ...(21) \\
\hline & \multirow{2}{*}{ Time } & Season & Spring, Summer, Fall, Winter (4) \\
\hline & & Day/Night & Day, Night (2) \\
\hline \multirow{2}{*}{$\begin{array}{l}\text { User } \\
\text { ence }\end{array}$} & - Cartoon Style & & Oriental, Western (2) \\
\hline & Exaggeration & & Exaggerated, Non-exaggerated (2) \\
\hline
\end{tabular}

scenario landmark events described in Table 1. Description of landmark events and cartoon images are annotated using the keywords in Table 2. For each landmark, similarities between cartoon character and background images are computed with user profile using the cartoon cut composition method. Computed similarities between a landmark event event $_{4}$ and some of cartoon character images are shown in Fig. 3. The cartoon images with the similarity over $50 \%$ are selected to be composed into cartoon cuts. These cartoon cuts are arranged into story stream graph as depicted in Fig. 4. Using story stream graph, final cartoon story is composed with cartoon cuts which do not violate the consistency constraints as shown in Table 3. With composed cartoon 


\begin{tabular}{|c|c|c|}
\hline \multicolumn{3}{|c|}{ Landmark Event } \\
\hline ID & Behavior & Environment \\
\hline 4 & Talk, Phone, Stand & University, Outdoor \\
\hline
\end{tabular}

\begin{tabular}{|c|c|}
\hline \multicolumn{2}{|c|}{ User Profile } \\
\hline Character Style & $\begin{array}{c}\text { Exaggeration } \\
\text { Preference }\end{array}$ \\
\hline Western & Exaggeration \\
\hline
\end{tabular}

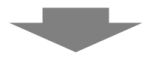

\begin{tabular}{|c|l|l|c|c|c|}
\hline \multicolumn{1}{|c|}{ Character Image Annotation } \\
\hline ID & \multicolumn{1}{|c|}{ Behavior } & Style & Exaggeration & Sim. \\
\hline 1000 & Talk, Phone, Stand & Oriental & Non-exaggerated & $42.8 \%$ & Image \\
\hline 1001 & Talk, Phone, Stand, Mad & Oriental & Exaggerated & $50 \%$ \\
\hline 1100 & Talk, Phone, Stand & Western & Non-exaggerated & $57 \%$ \\
\hline 1101 & Talk, Phone, Stand, Happy & Western & Exaggerated & $62.5 \%$ \\
\hline 2000 & Inspect, See & Oriental & Non-exaggerated & $0 \%$ & \\
\hline
\end{tabular}

Fig. 3. Semantic similarities between character images and the landmark event (ID =4)

\begin{tabular}{|l|}
\hline \multicolumn{1}{|c|}{$C C_{31}$} \\
\hline Eat, Sit, Western \\
\hline Restaurant, Indoor \\
\hline
\end{tabular}

\begin{tabular}{|l|}
$\mathrm{CC}_{42}$ \\
\hline $\begin{array}{l}\text { Phone, Stand, Western, } \\
\text { Exaggerated, Happy }\end{array}$ \\
\hline University, Outdoor \\
\hline
\end{tabular}

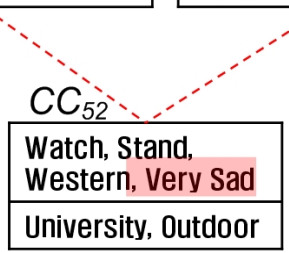

Fig. 4. A part of story streams generated by a landmark context example. Each box represents a cartoon cut which composed of annotations of the character image and the background image. Dashed lines represent the relation which violates the consistency constraints.

story, image representation of cartoon story is generated. Fig. 5 shows one of the composed cartoon story images. To evaluate the method, four composed cartoon story images were shown to participants. 
Table 3. A part of consistency constraints

\begin{tabular}{lll}
\hline ID & Preceding Behavior & Irrelevant Following Behavior \\
\hline 1 & Very Happy & Very Sad \\
2 & Happy & Very Sad \\
3 & Very Sad & Very Happy \\
4 & Very Sad & Happy \\
5 & Sad & Very Happy \\
\hline
\end{tabular}
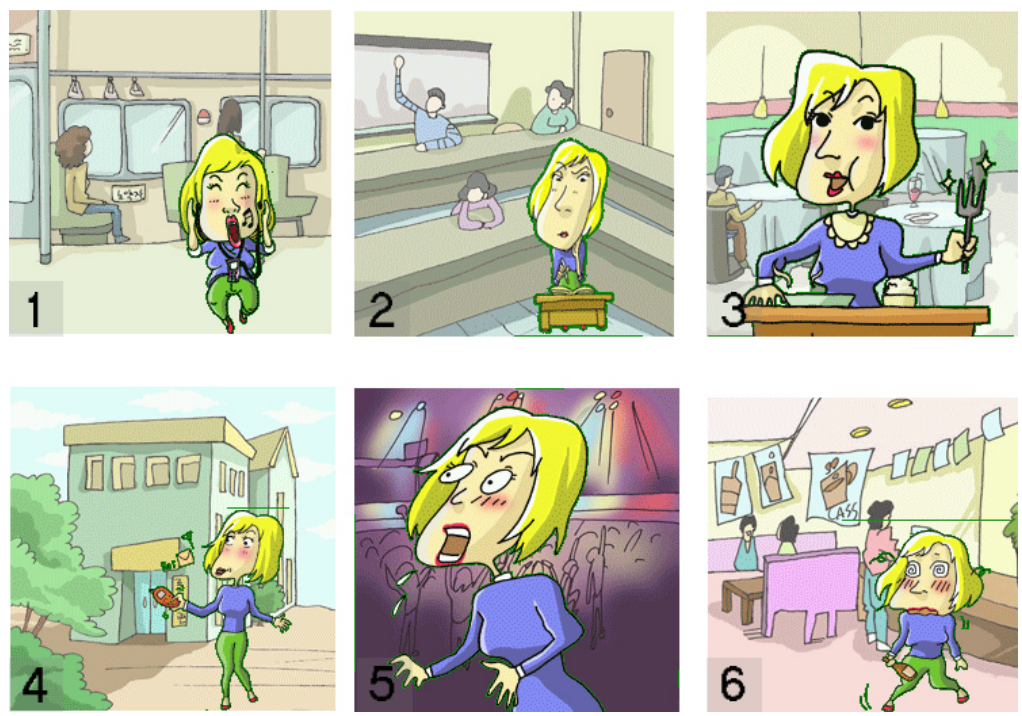

Fig. 5. A composed cartoon story from landmark examples in Table 1 to evaluate the diversity and the consistency

\subsection{Evaluation of Generated Cartoons}

By composed cartoon stories, user evaluation tests for cartoon cuts and cartoon story were performed with five participants. For each question, 5-point scale measure is used to grade an item. At first, cartoon cuts generated by a landmark example are evaluated in the criteria of the diversity and the descriptiveness. Fig. 6 shows the evaluation results. After cartoon cut evaluation, diversity and consistency of four cartoon stories were asked to the participants. The diversity of cartoon stories results in 4.0 of average and 1.0 of standard deviation, and the consistency of cartoon stories is depicted in Fig. 7. These data are not enough for the statistical significance, but the average score for each criterion shows affirmative tendencies. 


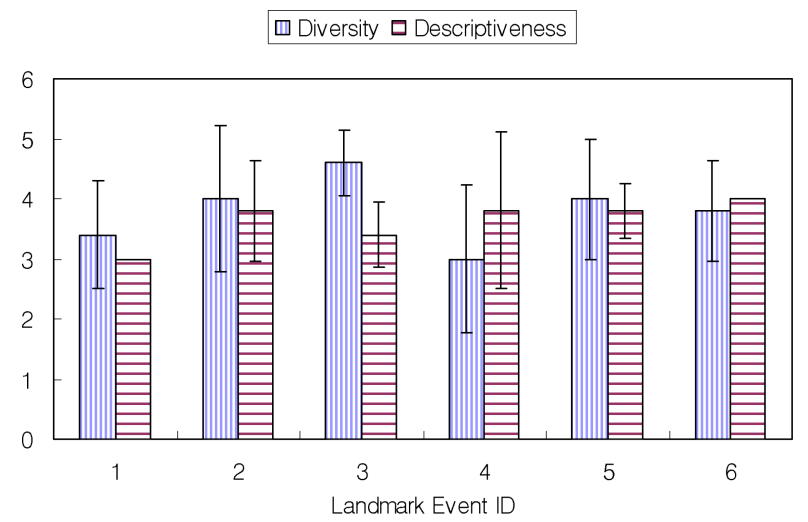

Fig. 6. Diversity and descriptiveness of cartoon cuts for each landmark event $(n=5)$

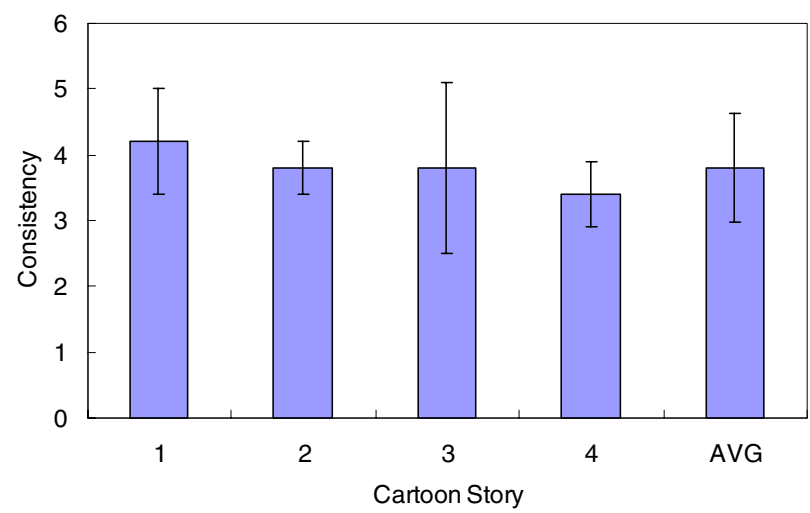

Fig. 7. Consistency Scores of four generated cartoon stories $(n=5)$

\section{Concluding Remarks}

To strengthen the effectiveness of the communication and the reflection of everyday mobile life, we generate funny cartoons with landmark events. From the landmark events and the user profile, we generate the cartoon cuts by applying semantic similarity between the context events and the images. Theses cartoon cuts are arranged into a cartoon story by using consistency constraints. By these methods, we can acquire a variety of funny cartoon stories with consistency. Because we use rather simple composition method, it can be smoothly used to compose the cartoons even in the limited mobile environments. But in this method, to exactly describe cartoon images, too much annotation costs are required to define cartoon images with a set of keywords. To decrease the annotation cost, cartoon image should be described with less keywords. By utilizing ontology like ConceptNet [11] or LifeNet [12], the annotation cost can be decreased. The approach with ontology can be also useful in selecting appropriate cartoon images and cartoon stories with landmark events. More fundamental 
approach to decrease annotation costs like automatic image annotation [13] will be more plausible. Our future works include ontology design for the cartoon image and landmark events annotation.

\section{References}

1. A. K. Dey, G. D. Abowd, and D. Salber, "A conceptual framework and a toolkit for supporting the rapid prototyping of context-aware applications," Human-Computer Interaction, vol. 16, no. 2, pp. 97-166, 2001.

2. R. Jain, "Multimedia electronic chronicles," IEEE Multimedia, vol. 10, no. 3, pp. 111-112, 2003.

3. R. Koster and W. Wright, A Theory of Fun for Game Design, Paraglyph Press, 2004.

4. R. Miikkulainen, "Script recognition with hierarchical feature maps," Connection Science, vol. 2, pp. 83-101, 1990.

5. E. Horvitz, S. Dumais, and P. Koch, "Learning predictive models of memory landmarks," CogSci 2004: 26th Annual Meeting of the Cognitive Science Society, 2004.

6. F. B. Viégas, D. Boyd, D. H. Nguyen, J. Potter, and J. Donath, "Digital artifacts for remembering and storytelling: Post history and social network fragments," Proc. of the 37th Hawaii International Conference on System Sciences, 2004.

7. Y. Sumi, R. Sakamoto, K. Nakao, and K. Mase, "ComicDiary: Representing individual experience in a comic style," UbiComp 2002, LNCS 2498, pp. 16-32, 2002.

8. A. W. M. Smeulders and A. Gupta, "Content-based image retrieval at the end of the early years," IEEE Trans. on Pattern Analysis and Machine Intelligence, vol. 22, no. 12, 2000.

9. M. Inoue and N. Ueda, "Retrieving lightly annotated images using image similarities," Proc. of the 2005 ACM Symposium on Applied Computing, pp. 1031-1037, 2005.

10. J. Cardoso and A. Sheth, "Semantic e-workflow composition," Journal of Intelligent Information Systems, vol. 21, no. 3, pp. 191-225, 2003.

11. H. Liu and P. Singh, "ConceptNet: A practical commonsense reasoning tool-kit," BT Technology Journal, vol. 22, no. 4, pp. 211-226, 2004.

12. P. Singh and W. Williams, "LifeNet: A propositional model of ordinary human activity," Distributed and Collaborative Knowledge Capture Workshop, 2003.

13. K. Barnard, P. Duygulu, D. Forsyth, N. de Freitas, D. M. Blei, and M. I. Jordan, "Matching words and pictures," Journal of Machine Learning Research, vol. 3, pp. 1107-1135, 2003. 